\title{
Next Generation Sequencing as an Emerging Technology in Rare Disease Genetics
}

\author{
Memoona Rasheed \\ Senior Lecturer, Department of Allied Health Sciences, Islamabad Medical and Dental College
}

The DNA sequencing techniques became available in 1970s. The technique developed by Sanger and colleague, referred to as Sanger Sequencing, became the most widely used method in DNA sequencing projects including the most famous Human Genome Project (HGP). ${ }^{1}$ The first draft human genome sequence was published separately by a public sector group, International Human Genome Sequencing Consortium (IHGSC), in Nature and by a private sector group, Celera Genomics, in Science in 2001. ${ }^{1}$ The estimated cost for HGP was 3 billion USD over a span of 15 years but this cost and time reduced to 300 million in 2 years due to the rapid technical advancements. ${ }^{2}$ In 2004, The National Human Genome Research Institute (NHGRI) started a funding program aimed to reduce the sequencing cost to 1000 USD which paved the path for development of the Next Generation Sequencing (NGS) technologies, also known as high throughput sequencing technologies. ${ }^{1}$

The first NGS technology was introduced by 454 Life Sciences (now Roche) in 2004 which utilized pyrosequencing method. Illumina sequencing platform and Sequencing by Oligo Ligation Detection (SOLiD) by Applied Biosystems (now Life Technologies) were commercialized in 2006 and 2007, respectively. ${ }^{3}$ Other NGS methods are also

\section{Correspondence:}

Memoona Rasheed

Email:memoona_rasheed@hotmail.com

Cite this Editorial: Rasheed M. Next Generation Sequencing as an Emerging Technology in Rare Disease Genetics. J Islamabad Med Dental Coll. 2020; 9(1):1-3.

Doi: 10.35787/jimdc.v9i1.521 available like Ion torrent, Pacific Bioscience and Oxford Nanopore but so far, Illumina holds the major market share due to availability of wide range of cost effective platforms. ${ }^{2}$ The Illumina's recently introduced NovaSeq 6000 system (www.illumina.com) has the capability of generation of 6 Terabyte data per run with fastest run time of 1-2 days with much reduced price but their promise of reducing sequencing cost per genome to 100 USD is still to be fulfilled.

The rapid drop in sequencing prices paved the path for clinical implementation of NGS technology. NGS has been used in cancer genomics, personalized medicine, forensic science, HLA typing and organ transplantation, clinical microbiology, prenatal diagnostics, new born screening and in identification of genetic players in rare disorders (Figure 1). Rare disorders, although individually uncommon but collectively affect millions of people world wide. These disorders occur due to mutations in single gene affecting its function and so far, 7000 rare disorders are known to occur with almost 15000 genes being involved. ${ }^{4}$ For molecular diagnosis of rare disorders, NGS based targeted gene panels are most commonly used for identification of mutation in genes already known to cause a disease. ${ }^{5}$ Whole exome sequencing (WES), which targets only protein coding portion of the genome ( $1 \%)$, is the second best option for identification of pathogenic mutations in already known and novel genes4 Identification of disease gene will help to unravel the molecular pathways which will be helpful in exploring therapeutic options. Currently, direct therapeutic approaches 
such as DNA replacement by gene therapy, protein /enzyme replacement therapy, transcriptional downregulation by antisense oligonucleotides or RNA interference and disease gene correction by using genome editing techniques are in clinical or pre-clinical trials. Beside these, rapid low cost and low risk therapies for rare diseases is also practiced such as dietary substrate omission or supplementation as in case of phenylketonuria, maple syrup urine disease and some other metabolic disorders. ${ }^{4}$

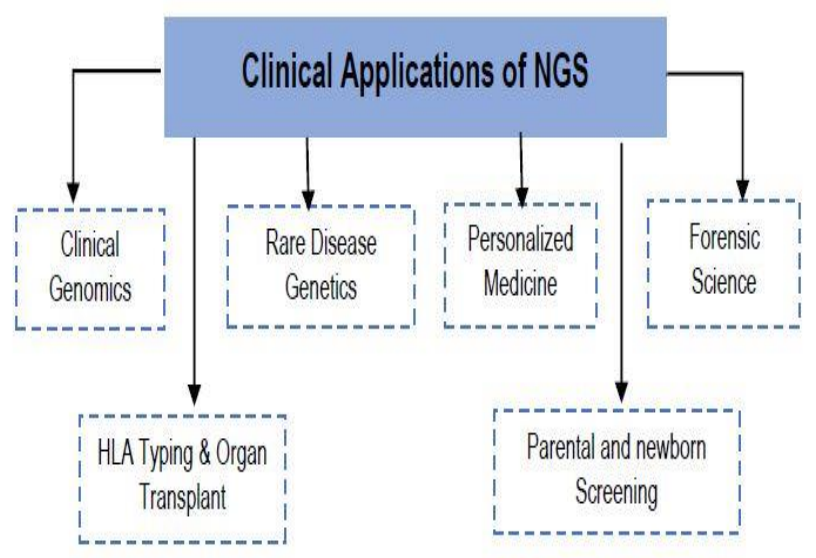

Figure 1: Clinical Applications of Next Generation Sequences

In Pakistan, 17-38\% marriages are consanguineous which have been long known to increase the risk of congenital disorders among children. ${ }^{6}$ Large scale genetic studies have been conducted on Pakistani families affected with various rare disorders by using next generation sequencing technologies which has led to identification of several novel genes and novel mutations in already known genes. Genetic analysis of 23 family affected with autosomal recessive primary microcephaly revealed that most of the disease-causing mutations were harbored by ASPM and WDR62.7 A study conducted on 60 families affected with Intellectual disability (ID) by using whole exome sequencing helped in identification of 30 novel candidate disease causing genes. $^{8}$

In another study, 192 ID affected consanguineous families, belonging to Pakistan and Iran were studied which helped in identification of 26 new ID causing genes. ${ }^{9}$ Targeted NGS on 12 Pakistani families affected with syndromic or non-syndromic hearing loss helped in identification of 8 novel disease causing variants in known genes. ${ }^{10}$ In another study, whole exome sequencing helped in developing molecular diagnosis for almost half of the cases in familial cohort inheriting retinal degeneration. ${ }^{11}$ Thus implementation of NGS technology in clinical practice for rare disease diagnosis by designing population specific gene panels or by whole exome sequencing are the two cost effective approaches which will not only shorten the time for disease diagnosis but will also help in deciding therapeutic options available to the patient.

\section{References}

1. Chan EY. Advances in sequencing technology. Mutat Res. 2005; 573(1-2): 13-40. Doi: 10.1016/j.mrfmmm.2005.01.004

2. Timmerman, L. DNA sequencing market will exceed $\$ 20$ billion, says Illumina CEO Jay Flatley. Forbes [online],https://www.forbes.com/sites/luketimmerm an/2015/04/29/qa-with-jay-flatley-ceo-of-illuminathe-genomics-company-pursuing-a-20bmarket/\#6b80ba6c42e7 (29 Apr 2015)

3. van Dijk EL, Auger $\mathrm{H}$, Jaszczyszyn $\mathrm{Y}$, Thermes $\mathrm{C}$. Ten years of next-generation sequencing technology. Trends Genet. 2014; 30(9): 418-26. Doi: 10.1016/j.tig.2014.07.001

4. Boycott KM, Vanstone MR, Bulman DE, MacKenzie $A E$. Rare-disease genetics in the era of nextgeneration sequencing: discovery to translation. Nat Rev Genet. 2013; 14(10): 681-91. Doi: $10.1038 / \mathrm{nrg} 3555$

5. Rehm HL. Disease-targeted sequencing: a cornerstone in the clinic. Nat Rev Genet. 2013; 14(4): 295-300. Doi: 10.1038/nrg3463

6. Hamamy $H$, Antonarakis SE, Cavalli-Sforza LL, Temtamy S, Romeo G, Kate LP et al. Consanguineous marriages, pearls and perils: Geneva International 
Consanguinity Workshop Report. Genet Med. 2011; 13(9): 841-47. Doi: 10.1097/GIM.0b013e318217477f

7. Wang R, Khan A, Han S, Zhang X. Molecular analysis of 23 Pakistani families with autosomal recessive primary microcephaly using targeted next-generation sequencing. J Hum Genet, 2017; 62(2): 299-304. Doi: 10.1038/jhg.2016.128

8. Riazuddin S, Hussain M, Razzaq A, Iqbal Z, Shahzad M, Polla DL, et al. Exome sequencing of Pakistani consanguineous families identifies 30 novel candidate genes for recessive intellectual disability. Molecular Psychiatry, 2017; 22(11): 1604-14. Doi: 10.1038/mp.2016.109

9. Harripaul R, Vasli N, Mikhailov A, Rafiq MA, Mittal K, Windpassinger $C$, et al. Mapping autosomal recessive intellectual disability: combined microarray and exome sequencing identifies 26 novel candidate genes in 192 consanguineous families. Mol Psychiatry. 2018; 23(4): 973-84. Doi: $10.1038 / \mathrm{mp} .2017 .60$

10. Wang R, Han S, Khan A, Zhang X. Molecular Analysis of Twelve Pakistani Families with Nonsyndromic or Syndromic Hearing Loss. Genet Test Mol Biomarkers. 2017; 21(5): 316-21. Doi: 10.1089/gtmb.2016.0328

11. Maranhao B, Biswas $P$, Gottsch ADH, Navani $M$, Naeem MA, Suk J, et al. Investigating the Molecular Basis of Retinal Degeneration in a Familial Cohort of Pakistani Decent by Exome Sequencing. PLoS One, 2015; 10(9): e0136561. Doi: 10.1371/journal.pone.0136561. 